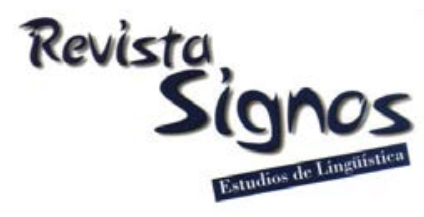

\title{
La comprensión de múltiples textos: Perspectivas y posibilidades para la investigación transdisciplinar
}

\author{
Multiple text comprehension: Perspectives and possibilities for \\ transdisciplinary research
}

\section{Moisés Damián Perales-} Escudero

UNIVERSIDAD DE QUINTANA ROO, UNIDAD CHETUMAL MÉXICO

mdperales@uqroo.edu.mx
Norma Alicia Vega López

UNIVERSIDAD AUTÓNOMA DE TAMAULIPAS MÉXICO navegalo@uat.edu.mx

Recibido: 17/XII/2015 / Aceptado: 27/V/2016

\section{Resumen}

La comprensión integrada de múltiples textos es una constante en la actividad académica y profesional; constituye una de las principales habilidades de alfabetización del Siglo XXI y supone la puesta en marcha de un conjunto de actividades de evaluación, integración y síntesis de información para la resolución de problemas. Develar, por un lado, los procesos psicolingüísticos subyacentes a estas actividades y, por otro, los recursos lingüístico-discursivos de la intertextualidad, han sido objetos de estudio por parte de diversas escuelas, como la teoría de múltiples documentos, la Teoría de la Valoración de la Lingüística Sistémico-Funcional y la polifonía enunciativa. No obstante, aún no se ha explorado de manera comparativa las particularidades y puntos de encuentro de estas tres perspectivas, ni tampoco las posibilidades que su integración ofrece para investigar la comprensión de múltiples textos desde una plataforma psicolingüística más compleja. Este trabajo tiene como objetivos comparar y contrastar estas tres escuelas, así como esbozar un programa de investigación transdisciplinar en esa materia. Para ello, revisamos y contrastatamos los fundamentos teóricos de las tres perspectivas así como los hallazgos, métodos y unidades de análisis más representativos de estudios de la comprensión de lectura inspirados en ellas, en especial aquellos de estudios hispanoamericanos. Finalmente, hacemos énfasis en las posibilidades que ofrece su integración reflexiva para avanzar en la comprensión de los fenómenos discursivos asociados a la comprensión de múltiples documentos citados dentro de un mismo texto, así como en los desafíos teórico-metodológicos que esto supone.

Palabras Clave: Comprensión de múltiples textos, Teoría de múltiples documentos, Teoría de la Valoración, polifonía enunciativa, consciencia metatextual. 


\begin{abstract}
Reading multiple texts in an integrated manner is a typical feature of twenty-first century academic and professional literacies; it involves a number of information evaluation, integration and synthesis activities. Different disciplines and schools of thought are involved in characterizing the cognitive processes behind these activities and the discursive resources of intertextuality. Among them are the Documents Model, Appraisal Theory - an offshoot of Systemic-Functional Linguistics -, and enunciative polyphony. However, these perspectives have not been examined contrastively despite the possibilities that their principled integration may offer for researching multiple text comprehension from a more psycholinguistical integrated manner. Therefore, this paper aims at comparing and contrasting these three schools and outlining a transdisciplinary research program on the matter. In order to accomplish this goal, we review and contrast the theoretical foundations and principal findings of the three perspectives, with special attention to Latin American studies. Finally, we outline possibilities for future research as well as theoretical and methodological challenges in furthering our understanding of the discursive phenomena associated with multiple text comprehension.
\end{abstract}

Key Words: Multiple text comprehension, the Documents Model, Appraisal Theory, enunciative poliphony, metatextual awareness.

\title{
INTRODUCCIÓN
}

Una de las principales características de las prácticas de lectura en el nivel superior es su naturaleza 'intertextual', pues aprender en las disciplinas demanda que alumnos comprendan e integren la información proveniente de múltiples textos en representaciones mentales coherentes, para realizar tareas de indagación, resolución de problemas, toma de decisiones, escribir ensayos, o iniciarse en el proceso de investigación a través de tesis de licenciatura, en algunos contextos. Estos procesos implican principalmente, la evaluación de la fiabilidad de las fuentes, la toma de postura, la explicación y construcción de argumentos (Britt \& Rouet, 2012). En el contexto universitario, es frecuente que estas tareas se lleven a cabo con base en textos que citan o aluden a una multiplicidad de otros textos, es decir, que contienen citas incrustadas.

Si bien estas demandas de lectura y escritura tienen el potencial para promover un aprendizaje profundo en las disciplinas, en ocasiones los estudiantes no se benefician de ellas si no van acompañadas de un proceso de enseñanza explícita de estrategias que los introduzca en las prácticas de alfabetización. La investigación ha puesto de relieve las dificultades que presentan los estudiantes para llevar a cabo procesos de comprensión a partir de múltiples textos, mismos que van desde poder explicar un fenómeno científico hasta argumentar las causas de un evento e identificar posturas opuestas en géneros diversos (Vega, 2011; Perales-Escudero, 2013a, 2013b). 
Estas dificultades se traducen en un doble desafío. Desde la práctica docente, es imperativo contar con propuestas de formación en la lectura disciplinar de múltiples textos. Este nos remite al segundo, a saber, la existencia diferentes perspectivas sobre la lectura intertextual, algunas de las cuales atienden más a las dimensiones psicológicas de ésta, y otras a las textuales. El objetivo de este trabajo es comparar y contrastar tres de estas perspectivas y, a partir de este contraste, esbozar un programa de investigación transdisciplinar integrado que permita arrojar luz sobre los fenómenos de comprensión de múltiples fuentes incluidas en un mismo texto. Las tres perspectivas analizadas son la teoría del modelo de documentos, la polifonía enunciativa y la teoría de la valoración de la Lingüística Sistémico-Funcional (LSF). Partimos del reconocimiento de que la primera persigue explícitamente la investigación de la comprensión de lectura, en tanto que las otras investigan primariamente fenómenos textuales pero han sido utilizadas en varios estudios focalizados en la comprensión. Puesto que nuestro propósito es la comparación contrastiva, en las siguientes secciones utilizamos puntualmente conceptos de una perspectiva para discutir o explicar los principios o hallazgos de las otras. Las perspectivas se presentan en el siguiente orden: el modelo de documentos, la polifonía enunciativa y la Teoría de la Valoración. En cada sección, se presentan sus postulados teórico-metodológicos y hallazgos principales. Tras ello, se contrastan sus divergencias y coincidencias teórico-metodológicas, acentuando las áreas de colaboración potencial. Finalmente, destacamos algunos de los desafíos y posibles beneficios que supondría la investigación transdisciplinar.

\section{El modelo de documentos}

Desde una perspectiva cognitiva la 'teoría del modelo de documentos', es un marco explicativo de los procesos de representación que lleva acabo el lector durante la comprensión de múltiples textos. Esta teoría tiene como fundamento el modelo de Construcción-Integración planteado por Kintsch (1998) entre otros, y asume que el procesamiento del texto resulta en la elaboración de una representación global coherente -modelo situacional-, derivada de la interacción entre el conocimiento previo del lector y el texto -texto base-, dirigido por los objetivos de lectura y las estrategias que el lector pone en marcha.

El uso del término 'documento', denota una categoría amplia que engloba diferentes géneros -artículo científico, libro- y formatos en que se comunica la información -impreso, digital- (Rouet, 2006). Una premisa central del modelo, es considerar un documento como una 'entidad' que el lector experimenta y se representa, esto es, un artefacto social que adopta determinadas 'formas' y 'funciones' -artículo científico, de opinión, web site- dentro de una comunidad discursiva, es decir, son escritos por alguien, en un contexto determinado, con un propósito específico para una audiencia meta (Britt, Rouet \& Braasch, 2013). 
El modelo de documentos señala que los lectores expertos inician primeramente 'experimentando' el documento razonando acerca de sus características, es decir, 'lo contextualiza' considerando al menos los siguientes aspectos: 1) La identidad del autor: Toman en cuenta el nombre, el estatus, motivaciones para escribir el texto y los grupos de trabajo a los que pertenece; 2) El contexto: consideran el lugar, el período histórico-cultural en el que fue creado y la reputación del medio de publicación del documento; 3) Respecto a la forma o características del texto, los lectores consideran el estilo del lenguaje y el tipo de texto; 4) Los objetivos retóricos del documento en términos de las intenciones del autor (ej. persuadir o explicar, entre otras) y la audiencia a la cual se dirige el texto (Perfetti, Rouet \& Britt, 1999).

A partir de dicha información el lector forma una representación denominada 'nodo de la fuente'. Asimismo, construye el significado del contenido de la fuente llamada 'nodo de contenido' a través de la elaboración de dos niveles de representación: el 'texto base', -construcción de un red de significados de la ideas a nivel micro y macroestructural- y el modelo situacional, se construye a partir de la integración del conocimiento previo del lector a la red de significados establecida en la base del texto (Kintsch, 1998). De acuerdo con Rouet (2006) ambos nodos (fuente y contenido) toman la forma de una red conceptual que integra el conocimiento previo que posee el lector sobre ellos y el conocimiento recién adquirido del texto, formando una representación o 'nodo de documento'. A su vez, ambos nodos se conectan a través de vínculos fuente-contenido, soportados por la consciencia que tiene el lector respecto a la información de la fuente y de su contenido semántico (Rouet, 2006; Strømsø, Bråten \& Britt, 2010).

La construcción de 'representaciones intertextuales' se realiza a partir del establecimiento de vínculos entre ambos nodos de distintos textos: 'vínculos fuentefuente y vínculos contenido-contenido' (Rouet, 2006). Los 'vínculos fuente-fuente' pueden ser establecidos mediante conexiones intertextuales a partir de la citación que realizan los autores de los textos con respecto a otros textos, pero también por el reconocimiento de sus 'credenciales' (ej. investigador, profesor), del 'estatus' que poseen dentro de un determinado campo de conocimiento (ej. investigador experto o novel) e incluso de las 'afiliaciones' a instituciones o asociaciones a las que se adhieren. También pueden establecerlos mediante conexiones basadas en la 'ubicación temporal' de la publicación de los diferentes textos y su correspondiente contexto histórico cultural (Strømsø, et al., 2010).

Por su parte, los 'vínculos contenido-contenido' que pueden establecer los lectores para construir representaciones intertextuales, no están del todo explicados. La evidencia empírica señala que dichas conexiones intertextuales pueden adoptar formas distintas dependiendo de una serie de condiciones como: (1) el tipo de tarea, si es explicativa o argumentativa (Wiley, Goldman, Graesser, Sanchez, Ash \& Hemmerich, 
2009); (2) las relaciones retóricas entre las fuentes utilizadas, por ejemplo, si éstas guardan una relación de 'complementariedad o contradicción', las cuales pueden ser inferidas a partir de la representación de las citas incrustadas; (3) el tipo de preguntas implicadas en la tarea -literales, profundas Wiley, Godman, Graesser, Sánchez, Ash y Hemmerich (2009), y el tipo de conocimiento asociado a éstas, por ejemplo, conocimiento causal o procedimental (Graesser, McNamara \& VanLehn, 2005) y (4) a las características de dominio de conocimiento disciplinar (ej. Historia, Biología, Geología). En síntesis, mediante la representación de cada documento (nodo fuente y nodo contenido) y de las representaciones intertextuales los lectores pueden construir un modelo de documentos más o menos integrado para responder a una determinada tarea de comprensión a partir de múltiples textos.

La teoría del modelo de documentos, se caracteriza por develar los mecanismos de procesamiento intertextual. Adopta como enfoque -aunque no exclusivo- estudios contrastivos 'experto-novel' y usa metodologías de corte experimental. Privilegia como unidad de análisis la cognición individual y grupal, y se interesa por los efectos sobre la comprensión de variables como el conocimiento previo y/o disciplinar, las condiciones de lectura y las estrategias de lectura. Entre los principales hallazgos, estudios pioneros y de frontera han indicado que el conocimiento previo del tema ${ }^{1} \mathrm{y}$ el dominio de la disciplina tienen un impacto en el uso de las fuentes y la comprensión intertextual. Por ejemplo, el estudio de Rouet, Favart, Britt y Perfetti (1997) indagó la influencia que tiene la pericia disciplinar de psicólogos e historiadores sobre el uso de estrategias heurísticas - corroboración, evaluación y contextualización- para evaluar la fuente y elaborar una opinión acerca de un hecho histórico. Los resultados mostraron que psicólogos e historiadores utilizaron de forma diferida la estrategia de evaluación, es decir, los historiadores poseían criterios más amplios para evaluar y utilizar las fuentes primarias de forma contrastiva en la construcción de su opinión. En términos generales, el dominio de la disciplina influye en formar una consciencia sobre la importancia de llevar a cabo procesos de evaluación de la fuente.

No obstante, estudios recientes señalan que algunos estudiantes universitarios no son conscientes de la importancia de la evaluación de las fuentes que les permita establecer vínculos fuente-contenido. Asimismo, señalan que para lograr una comprensión intertextual requieren de un alto conocimiento previo del tema, el cual les permite inferir las posturas complementarias y/o contradictorias (Gil, Bråten, Vidal-Abarca \& Strømsø, 2010; Kobayashi, 2014). Por su parte, Strømsø, Bråten, Britt y Ferguson (2013), que utilizaron como instrumento protocolos de pensamiento en voz alta, también señalan las dificultades que tienen los estudiantes para utilizar el conocimiento de la fuente en la evaluación e interpretación del contenido de ésta, además de mostrar una incipiente atención a las citas incrustadas en un solo texto. Este último aspecto ha recibido menos atención por parte de la investigación en esta 
perspectiva, que ha tendido a analizar la lectura de varios textos cortos de divulgación, sin citas incrustadas.

La teoría del modelo de documentos, se caracteriza por develar los mecanismos de procesamiento intertextual, adopta como enfoque -aunque no exclusivo- estudios contrastivos 'experto-novel'; utilizando metodologías de corte experimental, y como unidad de análisis el efecto que tiene el conocimiento previo y/o disciplinar, las condiciones de lectura y las estrategias. Entre los principales hallazgos estudios pioneros han indicado que el conocimiento previo del tema ${ }^{1}$ y el dominio de la disciplina tienen un impacto en el uso de las fuentes y la comprensión intertextual. Por ejemplo, el estudio pionero de Rouet et al. (1997) indagó la influencia que tiene la expertitud de la disciplina -psicólogos, historiadores- en el uso de estrategias heurísticas -corroboración, evaluación y contextualización- para evaluar la fuente y elaborar una opinión acerca de un hecho histórico. Los resultados mostraron que psicólogos e historiadores utilizaron de forma diferida la estrategia de evaluación, es decir, los historiadores poseían criterios más amplios para evaluar y utilizar las fuentes primarias de forma contrastiva en la construcción de su opinión. En términos generales, el dominio de la disciplina influye en formar una consciencia sobre la importancia de llevar a cabo procesos de evaluación de la fuente. No obstante, estudios recientes señalan que algunos estudiantes universitarios no son conscientes de la importancia de la evaluación de las fuentes que les permita establecer vínculos fuente-contenido. Asimismo, señalan que para lograr una comprensión intertextual requieren de un alto conocimiento previo del tema, que les permita inferir las posturas complementarias y/o contradictorias (Gil et al., 2010; Kobayashi, 2014). Por su parte el estudio de Strømsø et al. (2013), utilizando como instrumento protocolos de pensamiento en voz alta, también señala las dificultades que tienen los estudiantes para utilizar el conocimiento de la fuente en la evaluación e interpretación del contenido de ésta, además de mostrar una incipiente atención a las citas incrustadas en los textos.

Este último aspecto ha recibido menos atención por parte de la investigación desde el modelo de documentos. En un estudio novedoso y relevante, Bråten, Strømsø y Andreassen (2016) examinaron específicamente la lectura de citas incrustadas dentro de textos de divulgación que expresan varias posturas sobre temas controversiales relacionados con riesgos para la salud. Los participantes fueron estudiantes universitarios de diversas disciplinas. Una característica importante de este estudio fue la manipulación de aspectos textuales para incrementar la visibilidad de las fuentes. Esto se realizó de varias maneras, entra las cuales se contaron la ubicación de las fuentes en el texto, la atribución más o menos clara de la información a la fuente citada (es decir, presencia explícita de un locutor segundo con nombre como the investigation by Professor Torp vs. presencia de un sustantivo general como existing research.), y la manipulación de la representación de los temas (uso de celulares, uso de edulcorantes artificiales) como riesgosos o no para la salud. Este último aspecto se 
realizó lingüísticamente mediante la manipulación de verbos de decir con ACTITUD para infundirles diferentes valores de COMPROMISO. (existing research confirms... / existing research disproves...).

Se encontró que, en general los estudiantes realizaron muy pocos vínculos fuentecontenido y que la manipulación textual tuvo escasos efectos sobre ello. Por ejemplo, el uso de una fuente explícita (Professor Torp) en contraste con el uso de un sustantivo general (existing research) no favoreció la identificación de la fuente y su vinculación con el contenido. Una excepción a esta tendencia consistió en la mayor formación de vínculos fuente-contenido cuando el verbo de decir implicaba que el fenómeno discutido no presentaba riesgos para la salud. Otro hallazgo interesante lo constituye la confusión encontrada en la atribución de algunos contenidos al locutor primero o al locutor segundo. Este hallazgo converge con aquellos de las otras dos perspectivas revisadas líneas abajo.

\section{La polifonía enunciativa}

La polilfonía enunciativa es un abordaje para el análisis del discurso que tiene sus raíces en los trabajos de Bajtín, Benveniste, Ducrot y Mainguenau, entre otros. La polifonía enunciativa se inserta dentro de la rica tradición francesa de análisis del discurso y se enmarca dentro varias de sus preocupaciones centrales, como la otredad en el discurso, el discurso referido o citado, la determinación de lo social y lo individual en el interdiscurso y el intradiscurso y la textualización de aquél en éste. No es nuestro objetivo resumir ni discutir las diversas posturas y debates al respecto (Amossy, 2000; Montero, 2014), sino solo presentar aquellos de sus postulados que resultan más relevantes para este trabajo, a saber, aquellos que tienen que ver con la textualización de la otredad, como el sujeto enunciador, la heterogeneidad mostrada y el discurso referido.

Entre los postulados centrales de la polifonía enunciativa se encuentran la separación tripartita del sujeto hablante, del locutor y del enunciador, la cual se enraíza en el reconocimiento de la constitución discursiva del sujeto en el texto (Mainguenau, 1983; Ducrot, 1984). Aunque los distintos teóricos han ofrecido diversas definiciones y reconocido que las mismas no están exentas de problemáticas, en líneas generales y para los propósitos de este trabajo, podemos definir las distinciones entre sujeto, locutor y enunciador como sigue:

- Sujeto hablante: el emisor externo al texto, o autor con una existencia externa al discurso.

- Locutor: el autor textualizado, fuente de los enunciados de un texto; en el discurso referido se distingue el locutor primero del locutor segundo, correspondiendo el primero a quien cita y el segundo a quien es citado (Mainguenau, 1983). 
- Enunciador: posturas abstractas en el texto; Mainguenau (1983) puntualiza que, cuando los discursos de diferentes personajes (autores o conjuntos de ellos en los textos académicos) son referidos de manera indirecta, éstos se convierten en enunciadores.

Como apunta Maingueneau (1987), el locutor puede construir distintos niveles de representación de otras voces y de la propia en el discurso, y éste se constituye siempre en relación con otros discursos. En consecuencia, el papel de estos constructos en la lectura de múltiples textos no puede justipreciarse sin tomar en consideración otros constructos discursivos pertinentes, ya que los distintos locutores y enunciadores se constituyen en el marco de diversos discursos y los vocalizan. En ese sentido, resultan centrales las nociones de heterogeneidad y discurso citado o referido.

Sobre una base Lacaniana e interdiscursiva, Authier (1982, 1984) postula la heterogeneidad como una noción de orden más general con dimensiones textuales y extratuales, dentro de las cuales se enmarcaría la polifonía. Para Authier (1982), la presencia del otro es intrínseca a la psicología del sujeto; así también, el interdiscurso es constituyente del discurso. Usa el término 'heterogeneidad constitutiva' para capturar estos fenómenos, y postula que su textualización constituye la 'heterogeneidad mostrada'. La heterogeneidad mostrada hace referencia a las formas lingüísticas que señalan la presencia de la voz del otro. Esta última se divide en heterogeneidad marcada y no marcada. La primera nos remite a la textualización explícita de otras voces mediante el uso de elementos lingüísticos y tipográficos unívocos como el discurso directo, las comillas, las itálicas, o las glosas. La segunda nos remite a la textualización implícita sin interrumpir el flujo del discurso propio mediante, por ejemplo, el discurso indirecto libre, el pastiche, la ironía, la parodia o la paráfrasis y quizás también los distintos tipos de negación polifónica propuestos por Ducrot. Un planteamiento relevante para este trabajo es el de modalización autonímica, o juicio del locutor sobre su propia enunciación, especialmente cuando estos juicios evalúan discursos referidos.

La caracterización del discurso referido y su textualización es una preocupación focal de los estudios enunciativos $y$ ha sido tratada por muchos autores. Generalmente, se reconoce una división tripartita entre discurso directo, indirecto e indirecto libre. El discurso directo reproduce fielmente la vocalización de otro y la señala explícitamente la inserción de una vocalización del otro en el discurso propio mediante recursos sintácticos, léxicos y/o tipográficos (verba dicendi, comillas y otros, Maldonado, 1991). El discurso indirecto modifica y señala mediante recursos léxicos o sintácticos lo dicho por el otro pero mantiene su contenido proposicional. El discurso indirecto libre reproduce lo dicho por el otro sin identificar al locutor de manera explícita y sin los elementos lingüísticos o tipográficos de la citación (Maingueneau, 
1987). Al respecto, Maingueneau (1987) aporta la noción de discordancia, como una ruptura enunciativa que permite recuperar la presencia de discurso indirecto libre a partir de la identificación de dos planos de enunciación. Maingueneau y otros autores (Maldonado, 1991; Reyes, 1994) elucidan distintos tipos de discurso indirecto y sus posibles marcadores, como la oratio quasi oblicua o las citas encubiertas, cuya recuperación depende de elementos co-textuales y contextuales. También se ha propuesto la existencia de un estilo pseudo-directo (Maldonado, 1991), o indirecto subordinado (Reyes, 1984), que consiste en resumir un texto entrecruzando en el resumen fragmentos entrecomillados literales.

La Teoría de la Argumentación en la Lengua es otro aporte teórico que se relaciona con la polifonía enunciativa. Desde ella, se han producido numerosos aportes sobre el papel de diversos elementos lingüísticos en la textualización de argumentos que necesariamente implican la presencia de varias voces. Escapa a los alcances de este trabajo el hacer una caracterización exhaustiva de los mismos. Destacamos los constructos de discursos normativos y transgresores y su relación con familias de conectores discursivos (Ducrot, 2004), así como los hallazgos de García-Negroni, Marín y Hall (2005) sobre la polifonía en las nominalizaciones.

En resumen, los estudios enunciativos aportan descripciones finas de los recursos lingüísticos y tipográficos que permiten recuperar la presencia de otras voces y su papel en la argumentación. Un aporte distintivo lo constituye la posibilidad, desde los estudios enunciativos, de recuperar aspectos polifónicos aún en ausencia de marcadores lingüísticos unívocos. Esta posibilidad viene dada por elementos pragmáticos y contextuales que permiten establecer discordancias (Maingueneau, 1987). Otro aporte distintivo es la elucidación que hace del papel de marcas tipográficas en la textualización y posicionamiento de otras voces.

En los siguientes párrafos se revisan algunos estudios realizados desde la perspectiva enunciativa enfocados en la comprensión de lectura. Estos estudios se dividen en dos tipos: estudios textuales que buscan identificar lo que Marín y Hall (2003) denominan puntos críticos de incomprensión, y estudios empíricos que investigan el comportamiento de grupos de lectores con referencia a estos puntos de incomprensión.

Una línea de investigación muy valiosa en los estudios realizados desde la perspectiva de la polifonía enunciativa es la identificación y descripción de fenómenos microdiscursivos (por oposición a los fenómenos de organización textual) que pueden dificultar la comprensión. Tomando como unidad de análisis los segmentos microdiscursivos, estos estudios han realizado aportaciones valiosas para el estudio de la incomprensión. Por ejemplo, Hall y López (2011) analizan un corpus de manuales universitarios y encuentran que ciertas estrategias usadas para presentar las fuentes de manera colectiva e impersonal ('la teoría literaria', 'algunos críticos') así como el uso 
del pseudopasivo con 'se' ('se suele decir que...', 'se ha intentado definir') son comunes y pueden constituir puntos de incomprensión al difuminar al locutor. Como afirman las autoras, "el ocultamiento de las fuentes de enunciación produce ambigüedad y, por lo tanto, puede resultar una fuente de dificultad" (Hall \& Lopez, 2011: 637). En la misma línea, Hall y Marín (2011) analizan manuales universitarios en doce disciplinas y encuentran efectos similares de ambigüedad en la representación de la polifonía enunciativa. Hall (2011) contrasta la polifonía y argumentación de un manual con la de un artículo de investigación. Encuentra que, en el primero, las fuentes aparecen neutralizadas bajo el amparo de un sustantivo colectivo, lo cual provoca ambigüedad. En el segundo, las fuentes se citan minuciosamente pero ello también puede producir puntos de incomprensión.

Los estudios empíricos llevados a cabo desde esta perspectiva han sido de corte pre-experimental y han utilizado pruebas escritas para medir la comprensión en grupos de estudiantes. Por ejemplo, García-Negroni et al. (2005), identifican textualmente un punto de incompresión, el procesamiento de nominalizaciones polifónicas en el contexto de un discurso indirecto libre, y verifican si efectivamente obstaculiza la comprensión de una serie de pasajes que incluyen este tipo de nominalizaciones por parte de estudiantes universitarios argentinos. Encuentran que muy pocos de sus participantes fueron capaces de recuperar la complejidad enunciativa manifestada en un pasaje nominalizado, es decir, los puntos de vista involucrados. Concluyen llamando la atención sobre las complejidades y dificultades que entraña el procesamiento de estos elementos microdiscursivos.

Una serie de estudios referidos por Arnoux et al. (2002) ha puesto de manifiesto las dificultades que estudiantes pre universitarios y universitarios argentinos tienen para reconocer la atribución de segmentos textuales a voces distintas del locutor primero, comprender cuando un enunciado integra posturas en diversos niveles o planos de enunciación a lo largo del texto, así como la integración de representaciones proposicionales y enunciativas. Por su parte, el estudio de Aguilar y Fregoso (2013) con estudiantes universitarios mexicanos muestra que las dificultades para hacer conexiones fuente-contenido plausibles se relacionan con el formato de las citas (integradas vs. no integradas, citas en bloque, uso o no de comillas).

\section{La Teoría de la Valoración de la Lingüística Sistémico- Funcional}

La Teoría de la Valoración se compone de una serie de sistemas de semántica discursiva propuestos por la escuela de Sydney de la Lingüística Sistémico-Funcional; se inspira fuertemente en la polifonía Bajtiniana (Martin \& White, 2005) y comparte elementos comunes con el análisis del discurso francés. La Teoría de la Valoración: 
"elabora la noción de significado interpersonal y explora, describe y explica el modo en el que el lenguaje se usa para evaluar, adoptar posiciones, construir personas textuales y manejar posturas y relaciones interpersonales" (González, 2011: 109).

Como señala Kaplan (2004), la Valoración modela tres tipos de posicionamiento: el actitudinal o evaluativo, el dialógico y el intertextual. Los dos primeros son los ejes de la teoría y son modelados explícitamente en los subsistemas de la ACTITUD y el COMPROMISO respectivamente, en tanto que el último aparece subsumido en éstos ${ }^{2}$ El análisis de la Valoración trata de identificar los segmentos textuales que contienen instancia de evaluación (ACTITUD) y toma de posturas (COMPROMISO), clasificarlos conforme a las opciones sistémicas de la Valoración, y explicitar su contribución a la unidad textual local y global. Este último punto, distingue a la Teoría de la Valoración de la polifonía enunciativa.

En las secuencias de ACTITUD se identifica un emisor de la evaluación, o Valorador, un receptor, o Valorado, y un destinatario, o Valorante. El análisis LSF de Valoración se enfoca tanto en asignar estos roles semánticos al interior de cada secuencia de ACTITUD como en describir su distribución pautada a lo largo de segmentos textuales largos y/o textos completos (Martin \& White, 2005). También se describe la contribución de estas pautas a la unidad textual. Perales-Escudero y Sandoval-Cruz (en prensa) postulan que los roles de Valorador, Valorado y Valorante pueden ser ocupados por diversos locutores y enunciadores en el texto. Proponen, además, que la elucidación de las relaciones entre locutores/enunciadores y estos roles semánticos de la Valoración puede favorecer la identificación de posturas y contraargumentos durante la lectura retórica y crítica.

La red sistémica del COMPROMISO modela las relaciones dialógicas entre las distintas voces textualizadas en varios niveles de delicadeza. En el primero, se distingue entre aquellas vocalizaciones que establecen algún tipo de relación con otras voces (opciones de la heteroglosia, correspondientes a la polifonía) y aquellas que no lo hacen (aseveraciones categóricas, características de la monoglosia). En un segundo nivel, las opciones de la heteroglosia se dividen entre aquellas que abren el espacio dialógico a otras voces (expansión dialógica) o que lo cierran (contracción dialógica). En los niveles de mayor delicadeza, se modelan los tipos específicos de relaciones intertextuales consideradas desde la expansión dialógica (consideración, atribución [reconocimiento, distanciamiento]) y la contracción dialógica (refutación [negación, contraexpectativa], proclamación [coincidencia, pronunciamiento, respaldo]). Así, la Teoría de la Valoración aporta una manera sistemática de analizar las relaciones retóricas de las fuentes citadas o aludidas dentro de un mismo texto.

El abordaje de los fenómenos de la compresión de lectura desde la LSF se ha centrado sobre todo en el análisis y modelado del discurso áulico, el cual ha sido la unidad de análisis en la mayoría de los estudios teóricos y empíricos (Rose, 2007; Rose 
\& Martin, 2012). Los estudios han sido de corte intervencionista y llevan la impronta de la psicología sociocultural vygotskyana. Aunque son numerosos los trabajos que describen el discurso de los libros de texto escolares, estos no han puesto el acento sobre la identificación de puntos críticos de incomprensión. En general, la mayoría de los estudios LSF realizados por autores anglosajones evitan referirse a aspectos de la cognición de los lectores.

Sin embargo, algunos estudios recientes realizados en México en contextos de inglés L2 han incursionado en los aspectos sociocognitivos y metacognitivos de la comprensión lectora aislado y atendido puntos de incomprensión a partir del análisis del discurso áulico en el contexto de intervenciones. Por ejemplo, Perales-Escudero (2011, 2013a, 2013b) encuentra que lectores universitarios mexicanos no identifican adecuadamente el Valorador, Valorado y Valorante en secuencias intertextuales en las que el locutor vocaliza diversas evaluaciones a través de varios locutores segundos, enunciadores y puntos de vista. Ello ocasiona dificultades para identificar posturas en artículos periodísticos de opinión política en inglés L2. Estos hallazgos convergen con aquellos de los estudios polifónicos en identificar la inserción de evaluación en el discurso referido (evaluación presente en verbos de decir, evaluación de las evaluaciones de otros) como fuentes de dificultades para la construcción de representaciones intertextuales. Perales-Escudero, Reyes y Hernández, (2015) investigaron el papel de conocimientos sobre el lenguaje valorativo y actividades metalingüísticas derivadas de la Teoría de la Valoración sobre la comprensión inferencial y metacognición de estudiantes universitarios mexicanos durante la lectura de artículos de investigación en lingüística aplicada en inglés L2. Encontraron que las actividades metalingüísticas favorecen la comprensión e integración de argumentos provenientes de varias fuentes citadas e inciden favorablemente sobre algunos procesos de metacomprensión.

Un elemento clave de estos estudios mexicanos inspirados en la Teoría de la Valoración es el uso de organizadores discursivos como instrumentos de mediación en la construcción interpsicológica de conocimientos metatextuales y de estrategias de lectura de múltiples fuentes en textos argumentativos. Aquí, es necesario distinguir los organizadores gráficos de los organizadores discursivos. Los primeros remiten a la estructura global del texto completo o de secciones del mismo y se relacionan con las estructuras textuales (Jiang \& Grabe, 2007). Los segundos, propios de la LSF, promueven el procesamiento semántico de segmentos micro-discursivos locales de manera enlazada a lo largo de una sección o texto completo. Su finalidad es contribuir a la formación de modelos mentales integrados del texto a partir de a) la recuperación congruente de los roles semánticos en segmentos locales la Valoración por parte de los lectores, los cuales incluyen la adscripción de evaluaciones a los distintos locutores y enunciadores en distintos planos enunciativos $\mathrm{y}, \mathrm{b}$ ) la focalización de la atención en 
las pautas de enunciación y unidad textual creada por la repetición y variación de estos roles semánticos a lo largo del texto. En otras palabras:

“... el llenado del organizador discursivo permite al lector reconstruir la pauta de significados interpersonales que dan unidad a las distintas secciones del texto al presentar posturas contrapuestas. Al mismo tiempo, permite discernir entre la evaluación del autor y la de otras voces" (Perales-Escudero \& Sandoval-Cruz, en prensa).

Mediante estudios ecológicos del discurso áulico y estudios cuasi-experimentales con lectores universitarios (con el grupo de lectores como unidad de análisis), se ha encontrado que el análisis de pautas de unidad textual de ACTITUD y de COMPROMISO asistido por organizadores discursivos contribuye a la construcción de representaciones enunciativas o modelos intertextuales plausibles, tanto en géneros periodísticos (Perales-Escudero, 2011) como académicos (Perales-Escudero et al., 2015) leídos en inglés L2.

\section{Una propuesta de integración para el estudio transdiciplinar de la comprensión de textos con citas incrustadas}

En esta sección se contrastan las tres perspectivas (ver Tabla 1). Nuestro planteamiento base es que una mayor integración de las dos perspectivas lingüísticas y la perspectiva psicológica revisadas puede resultar fructífera para nuestro entendimiento de los fenómenos de comprensión intertextual y para el desarrollo de intervenciones didácticas, específicamente en la lectura de textos académicos que incluyen múltiples citas a otros documentos, o citas incrustadas. En ese sentido, la teoría del modelo de documentos ha considerado muy escasamente el papel de las variables textuales y de conocimiento metatextual, en tanto que las dos perspectivas lingüísticas han prestado poca atención al papel de aspectos como el conocimiento previo del tema, de las fuentes, y la estructuración de estos conocimientos en la cognición.

Tabla 1. Síntesis comparativa de las perspectivas de investigación de la lectura intertextual.

\begin{tabular}{|l|l|l|l|}
\hline & \multicolumn{1}{|c|}{$\begin{array}{c}\text { Modelo de } \\
\text { documentos }\end{array}$} & \multicolumn{1}{|c|}{ Polifonía enunciativa } & \multicolumn{1}{|c|}{$\begin{array}{c}\text { Lingǘstica } \\
\text { sistémica } \\
\text { funcional }\end{array}$} \\
\hline $\begin{array}{l}\text { Enfoque } \\
\text { metodológico }\end{array}$ & $\begin{array}{l}\text { Estudios } \\
\text { experimentales- } \\
\text { laboratorio }\end{array}$ & $\begin{array}{l}\text { Identificación de puntos de } \\
\text { incomprensión } \\
\text { Estudios pre- } \\
\text { experimentales }\end{array}$ & $\begin{array}{l}\text { Estudios } \\
\text { ecológicos-áulicos } \\
\text { Estudios cuasi- } \\
\text { experimentales }\end{array}$ \\
\hline $\begin{array}{l}\text { Unidades de } \\
\text { análisis }\end{array}$ & $\begin{array}{l}\text { Cognición individual y } \\
\text { grupal }\end{array}$ & $\begin{array}{l}\text { Segmentos } \\
\text { microdiscursivos } \\
\text { Cognición grupal }\end{array}$ & $\begin{array}{l}\text { Discurso áulico } \\
\text { Segmentos de } \\
\text { VALORACION y } \\
\text { su contribución a la } \\
\text { unidad textual }\end{array}$ \\
\hline
\end{tabular}




\begin{tabular}{|c|c|c|c|}
\hline & & & Cognición grupal \\
\hline $\begin{array}{l}\text { Variables } \\
\text { consideradas } \\
\text { o controladas }\end{array}$ & $\begin{array}{l}\text { Niveles de } \\
\text { comprensión: } \\
\text { intratextual e } \\
\text { intertextual } \\
\text { Conocimiento previo } \\
\text { del tema, de la fuente y } \\
\text { de estrategias } \\
\text { Uso de la fuentes } \\
\text { Pericia disciplinar }\end{array}$ & $\begin{array}{l}\text { Estatus de lector inexperto } \\
\text { (considerado sin control } \\
\text { estadístico) }\end{array}$ & $\begin{array}{l}\text { Conocimiento } \\
\text { previo } \\
\text { (considerado sin } \\
\text { control estadístico) }\end{array}$ \\
\hline Instrumentos & $\begin{array}{l}\text { Pruebas de verificación } \\
\text { de sentencias } \\
\text { Pruebas de } \\
\text { transferencia } \\
\text { Protocolos de } \\
\text { pensamiento en vOz } \\
\text { alta } \\
\text { Pruebas escritas } \\
\text { (explicativas y } \\
\text { argumentativas) } \\
\text { Textos cortos }\end{array}$ & $\begin{array}{l}\text { Pruebas escritas } \\
\text { Textos completos (estudios } \\
\text { textuales) } \\
\text { Fragmentos de textos } \\
\text { (estudios pre- } \\
\text { experimentales) }\end{array}$ & $\begin{array}{l}\text { Organizadores } \\
\text { discursivos } \\
\text { Pruebas escritas } \\
\text { Textos completos }\end{array}$ \\
\hline
\end{tabular}

Entonces, un área de oportunidad que presentan los estudios psicológicos realizados desde el modelo de documentos es la ausencia casi total de una consideración explícita del impacto de la presencia y procesamiento de elementos lingüísticos y estilísticos concretos, así como de la consciencia del lector acerca de ellos, sobre la construcción de representaciones intertextuales a partir de las citas incrustadas. Es decir, no se ha considerado el posible papel compensatorio que puede jugar la conciencia metatextual (conocimiento de la organización esquemática, pautas lingüísticas microdiscursivas de la heterogeneidad mostrada, tipos de discurso referido, recursos tipográficos) en la formación de modelos intertextuales a partir de citas incrustadas, especialmente en lectores en procesos de iniciación disciplinar que no pueden usar conocimientos previos para guiar su comprensión. Con excepción de Strømsø et al. (2013) y Bråten et al. (2016), no encontramos otros estudios que exploren los procesos o el tipo de conocimiento discursivo que utilizan los lectores para representarse las referencias incrustadas en el texto, p.ej. en géneros como el reporte de investigación y la revisión de literatura- y el papel que éstas juegan en la formación de vínculos-fuente-contenido y contenido-contenido.

Las dos perspectivas lingüísticas ofrecen posibilidades para avanzar un programa de investigación transdisciplinar en ese sentido. Por ejemplo, las opciones sistémicas del COMPROMISO y los postulados y hallazgos de los estudios enunciativos (p.ej. aquellos referidos a la textualización de locutores y enunciadores, la heterogeneidad mostrada, los tipos de discurso referido, sus marcas sintácticas y tipográficas, el papel de los marcadores discursivos y estilísticos en la argumentación polifónica) pueden proporcionar una descripción detallada de las relaciones retóricas de oposición y 
complementariedad y de los vínculos fuente-fuente y contenido-contenido. Estos últimos constructos son postulados desde el modelo de documentos, pero su descripción en el estado actual de la teoría es bastante superficial. Esta descripción minuciosa podría permitir realizar estudios experimentales con una mejor caracterización de las variables textuales que inciden en la formación de representaciones intertextuales, así como con una manipulación más fina de los textos que la realizada por estudios como el de Bråten et al. (2016), la cual es aún incipiente. Ello posibilitaría una caracterización más detallada y profunda de los procesos psicolingüísticos de los lectores.

Por otro lado, el modelo de documentos ofrece la posibilidad de construir instrumentos precisos y confiables para caracterizar las representaciones intertextuales y para atender el papel de otras variables, como el conocimiento previo del tema, de las fuentes, y su naturaleza integrada o dispersa, en la formación de estas representaciones. Asimismo, estos métodos experimentales pueden permitir verificar la aplicabilidad de los puntos críticos de incomprensión identificados por los estudios enunciativos con diversos grupos de lectores. Por ejemplo, los hallazgos de Bråten et al. (2016) parecen confirmar las predicciones de Hall y López (2011) y Hall (2011) en el sentido de que tanto el uso de sustantivos colectivos como concretos para referirse a las fuentes constituyen puntos de incomprensión.

A diferencia de la perspectiva polifónica, los estudios LSF no han considerado el papel de los aspectos tipográficos y estilísticos, por lo que en ellos se ubica un área de oportunidad para la investigación transdiciplinar. Por otro lado, la orientación explícita de la LSF hacia la identificación de roles semánticos de la evaluación en los niveles textuales local y global, así como su enfoque intervencionista vygotskyano, pueden complementar la perspectiva polifónica y la del modelo de documentos, tanto para la descripción de la interacción lector-texto-contexto como para el desarrollo de tareas e instrumentos de investigación y de intervenciones áulicas con textos que incluyen referencias incrustadas. En el primer sentido, el análisis de Valoración mediante organizadores discursivos parece prometedor para asistir la identificación de las voces del locutor primero y segundo, y los diversos enunciadores o posturas argumentativas, así como su continuidad o variación a lo largo del texto y en diversos tipos de discurso referido. El organizador discursivo podría, entonces, utilizarse para intervenir sobre los puntos críticos de incomprensión identificados por los estudios enunciativos y del modelo de documentos.

En el segundo sentido, desde el modelo de documentos podría investigarse de manera controlada el impacto de la identificación de roles semánticos valorativos mediante organizadores discursivos de la Valoración en la formación de nodos intertextuales en conjunto con otras variables: conocimiento previo, tipo de tarea, tipo de género y conocimiento metatextual. La adopción explícita de la terminología de identificación de voces de la polifonía enunciativa y de relaciones retóricas del 
COMPROMISO podría permitir también una descripción más fina de la naturaleza de los procesos que median la formación de nodos de documentos, fuente-fuente y contenido-contenido, en situaciones de lectura donde los lectores no tienen un conocimiento previo amplio y estructurado, como es el caso de los estudiantes de educación superior.

La línea de investigación sobre los organizadores discursivos de la LSF se beneficiaría de la inclusión de los aspectos tipográficos y estilísticos, así como de otros puntos de incomprensión identificados por los estudios enunciativos relativos a los tipos de discurso referido, particularmente las disonancias que permiten identificar la presencia de discurso indirecto libre en ausencia de marcas unívocas. También se beneficiaría del uso de métodos propios del modelo de documentos como las pruebas de verificación de sentencias intertextuales y de la estructuración de conocimientos previos. Este último aspecto podría aplicarse a la estructuración de los conocimientos metatextuales de los lectores, tema inexplorado desde el modelo de documentos pero implícito en las otras dos perspectivas, las cuales, sin embargo, no abordan sus dimensiones cognoscitivas o lo hacen de manera incipiente.

\section{CONCLUSIONES}

A lo largo del artículo hemos señalado y contrastado las aportaciones de las teorías revisadas. También hemos señalado puntos de cruzamiento y de colaboración potencial entre ellas. El modelo de documentos nos brinda un marco explicativo de los procesos de representación que lleva a cabo un lector ante la comprensión de múltiples textos. Señala la influencia que tiene el conocimiento estructurado de la disciplina en las representaciones intertextuales que establece el lector a nivel de fuente y de contenido. No obstante, esta evidencia se deriva principalmente de estudios experimentales, en situaciones de lectura controladas, es decir, con textos de corta extensión y principalmente de divulgación científica y de opinión.

Por su parte, la polifonía enunciativa y la Teoría de la Valoración nos ofrecen elementos estilístico-discursivos para operacionalizar la representación de las referencias incrustadas en el texto base y medir su efecto en la comprensión intertextual, así como un instrumento de intervención, el organizador discursivo de la Valoración. Los estudios realizados desde estas dos perspectivas en general se caracterizan por su validez ecológica, un menor control de variables y un mayor uso de textos académicos universitarios auténticos. Entonces, algunos desafíos metodológicos importantes para la realización de estudios transdisciplinares tienen que ver con el aseguramiento de la validez ecológica en cuanto al uso del tipo y extensión de los textos, que permita medir los niveles de comprensión en situaciones de lectura más reales, fuera del ambiente controlado de un laboratorio. Ello puede, sin embargo, dificultar el control de variables como el nivel conocimiento previo de los lectores y su 
estructura, la naturaleza y tiempo de la tarea, la pericia disciplinar y la consciencia metatextual de los lectores. Estas y otras dificultades compelen a los investigadores a la reflexión sobre aspectos tales como las bases epistémicas del paradigma nomotético y del interpretativo, la naturaleza y los alcances del conocimiento que éstos producen separadamente y en conjunto, y las oportunidades y limitaciones que cada uno ofrece.

No obstante estos retos, creemos que la investigación transdiciplinar ofrece oportunidades prometedoras para indagar el papel que juega el conocimiento metatextual en la representación de las fuentes y la comprensión intertextual en ausencia de un conocimiento previo profundo del tema. Los resultados de este tipo de investigación permitirían orientar el diseño de intervenciones en el aula e incidir en el aprendizaje disciplinar a través de la lectura. El esfuerzo que implica la investigación transdisciplinar podría, en consecuencia, producir resultados valiosos para la teoría y para la práctica docente. 


\section{REFERENCIAS BIBLIOGRÁFICAS}

Aguilar, L. E. \& Fregoso, G. (2013). La lectura de la polifonía e intertextualidad en el texto científico. Revista mexicana de investigación educativa, 18(57), 413-435.

Amossy, R. (2000). L'argumentation dans le discours. París: Nathan.

Arnoux, E., Nogueira, S. \& Silvestri, A. (2002). La construcción de representaciones enunciativas: El reconocimiento de voces en la comprensión de textos polifónicos. Revista Signos. Estudios de Lingüística, 35(52), 129-148.

Authier, J. (1982). Hétérogénéité montrée et hétérogénéité constitutive: Éléments pour une approche de láutre dans le discours. $D R L A, 26,91-151$

Authier, J. (1984). Hétérogénéité(s) énonciative(s). Langages, 73, 108-109.

Bråten, I., Strømsø, H., y Andreassen, R. (2016). Sourcing in profesional education: do text factors make a difference? Reading and Writing. [en línea]. Disponible en: http:/ / link.springer.com/article/10.1007\%2Fs11145-015-9611-y

Britt, M. A. \& Rouet, J.-F. (2012). Learning with multiple documents: Component skills and their acquisition. En J. R. Kirby \& M. J. Lawson (Eds.), Enhancing the quality of learning: Dispositions, instruction, and learning processes (pp. 276-314). Nueva York, NY: Cambridge University Press.

Britt, M. A., Rouet, J.-F. \& Braasch, J. (2013). Documents as entities: Extending the situation model theory of comprehension. En M. A. Britt, S. R. Goldman \& J.-F. Rouet (Eds.), Reading: from words to multiple texts (pp. 160-179). Nueva York: Routledge.

Ducrot, O. (1984). Le dire et le dit. Les Éditions de Minuit: París.

Ducrot, O. (2004). Sentido y argumentación. En E. Arnoux \& M. M. García Negroni (Eds.), Homenaje a Oswald Ducrot (pp. 359-370). Buenos Aires: Eudeba.

García Negroni, M. M., Marin, M. \& Hall, B. (2005). Ambigüedad, abstracción y polifonía del discurso académico: Interpretación de las nominalizaciones. Revista Signos. Estudios de Lingüística, 38(57), 49-60.

Gil, L., Bråten, I., Vidal-Abarca, E. \& Strømsø, H. I. (2010). Summary versus argument tasks when working with multiple documents: Which is better for whom? Contemporary Educational Psychology, 35,157-173.

González, M. (2011). La expresión lingüística de la actitud en el género de opinión: el modelo de la valoración. Revista de Lingüistica Teórica y Aplicada, 49, 109-141. 
Graesser, A. C., McNamara, D. S. \& VanLehn, K. (2005). Scaffolding deep comprehension strategies through point\&query, autotutor, and iSTART. Educational Psychologist, 40(4), 225-234.

Hall, B. (2011). Efectos de neutralidad: El caso de los manuales universitarios. En M. M. García-Negroni (Coord.), Los discursos del saber: Prácticas discursivas y enunciación académica (pp. 133-150). Buenos Aires: Editoras del Calderón.

Hall, B. \& López, M. I. (2011). Discurso académico: Manuales universitarios y prácticas pedagógicas. Literatura y Lingüística, 23, 167-192.

Hall, B. \& Marín, M. (2011). El discurso académico: Complejidad discursiva. En M. M. García-Negroni (Coord.), Los discursos del saber: Prácticas discursivas y enunciación académica (pp.101-132). Buenos Aires: Editoras del Calderón.

Jiang, X. \& Grabe, W. (2007). Graphic organizers in reading instruction: Research findings and issues. Reading in a Foreign Language, 19, 34-55.

Kaplan, N. (2004). Nuevos desarrollos en el estudio de la evaluación en el lenguaje: La Teoría de la Valoración. Boletín de Lingüística, 52-78 [en línea] Disponible en: http:/ / redalyc.org.www.redalyc.org/articulo.oa?id=34702203

Kintsch, W. (1998). Comprehension: A paradigm for cognition. Nueva York: Cambridge University Press.

Kobayashi, K. (2014). Students' consideration of source information during the reading of multiple texts and its effect on intertextual conflict resolution. Instructional Science, 42(2), 183-205.

Lemke, J. L. (1985). Ideology, intertextuality, and the notion of register. Systemic Perspectives on Discourse, 1, 275-294.

Maingueneau, D. (1983). Sémantique de la polémique. L'Age de l'homme: Lausanne.

Maingueneau, D. (1987). Nouvelles tendences en analyse du discours. París: Hachette.

Maldonado, C. (1991). Discurso directo y discurso indirecto. Madrid: Taurus Universitaria.

Marín, M. \& Hall, B. (2003). Los puntos críticos de incomprensión de la lectura en los textos de estudio. Lectura y Vida. Revista Latinoamericana de Lectura, 24, 22-29.

Martin, J. R. \& White, P. R. R. (2005). The language of evaluation: Appraisal systems in English. Nueva York: Palgrave Macmillan.

McNamara, D. S., Kintsch, E., Songer, N. B. \& Kintsch, W. (1996). Are good texts always better? Text coherence, background knowledge, and levels of understanding in learning from text. Cognition and Instruction, 14, 1-43. 
McNamara, D. S. \& O’Reilly, T. (2010). Theories of comprehension and comprehension skill: Knowledge and strategies versus capacity and suppression. En A. M. Columbus (Ed.), Advances in Psychology Research (pp. 113-136). Hauppauge, NY: Nova Science Publishers.

Montero, A. S. (2014). El análisis del discurso francés y el abordaje de las voces ajenas: Interdiscurso, polifonía, heterogeneidad y topos. En M. Canales (Comp.), Escucha de la escucha. Análisis e interpretación en la investigación cualitativa (pp. 2550). Santiago de Chile: LOM-FLACSO.

Perales-Escudero, M. D. (2011). Teaching and learning critical reading at a Mexican university: An emergentist case study. Unpublished doctoral dissertation, University of Michigan, Ann Arbor, EUA.

Perales-Escudero, M. D. (2013a). Teaching intercultural awareness in the EFL classroom: A case study using critical reading. Intercultural Education, 24(3), 251-263.

Perales-Escudero, M. D. (2013b). Attractor states, control parameters and coadaptation in L2 inferential comprehension: $A$ design-based study. Revista Brasileira de Linguistica Aplicada, 13(2), 463-492

Perales-Escudero, M. D., Reyes, M. \& Hernández, E. (2015). Impacto de una intervención lingüística en la comprensión inferencial retórica y la metacognición durante la lectura académica en inglés lengua extranjera: Un estudio cuasi-experimental con métodos mixtos. Revista Signos. Estudios de Lingüistica, 48(89), 332-354.

Perales-Escudero, M. D. \& Sandoval-Cruz, R. I. (en prensa). La lectura retórica desde la enseñanza discursivo-cognitiva: Una alternativa para la alfabetización académica. En G. Bañales-Faz, M. Castelló \& N. Vega-López (Coords.), Enseñar a leer y escribir en la educación superior. Propuestas educativas basadas en la investigación. Ciudad de México: Editorial SM.

Perfetti, C. A., Rouet, J.-F. \& Britt, M. (1999). Toward a theory of documents representation. En H. van Oostendorp \& S. R. Goldman (Eds.), The construction of mental representations during reading (pp. 99-122). Mahwah, NJ: Erlbaum.

Reyes, G. (1984). Polifonía textual. Madrid: Gredos.

Reyes, G. (1994). Los procedimientos de citas: Citas encubiertas y ecos. Madrid: Arco Libros.

Rose, D. (2007). A reading based model of schooling. Pesquisas em Discurso Pedagógico, 4(2) [en línea] Disponible en: http://www.maxwell.lambda.ele.puc-rio.br 
Rose, D. \& Martin, J. R. (2012). Learning to write, reading to learn: Genre, knowledge and pedagogy in the Sydney school. Sheffield: Equinox Publishing.

Rouet, J.-F. (2006). The skills of document use: From text comprehension to Webbased learning. Mahwah, NJ: Erlbaum.

Rouet, J.-F., Favart. M., Britt, M. \& Perfetti C. A. (1997). Studying and using multiple documents in history: Effects of discipline expertise. Cognition and Instruction, 15(1), 85-106.

Strømsø, H. I., Bråten, I. \& Britt, M. A. (2010). Reading multiple texts about climate change: The relationship between memory for sources and text comprehension. Learning and Instruction, 20(3), 192-204.

Strømsø, H., Bråten, I., Britt, M. A. \& Ferguson, L. (2013). Spontaneous sourcing among students reading multiple documents. Cognition and Instruction, 31(2), 176-203.

Vega, N. (2011). Comprensión de múltiples textos expositivos: Relaciones entre conocimiento previo y autorregulación. Tesis doctoral, Universitat Ramon Llull, Barcelona, España.

Wiley, J., Goldman, S. R., Graesser, A. C., Sánchez, C. A., Ash, I. \& Hemmerich, J. (2009). Source evaluation, comprehension, and learning in Internet science inquiry tasks. American Educational Research Journal, 46(4), 1060-1106.

\section{NOTAS}

${ }^{1}$ Estudios acerca de la comprensión intratextual, han señalado el papel que desempeña el conocimiento previo del tema -alto y bajo- en relación con otras variables como la cohesión y las habilidades lectoras, en la formación de modelos situacionales. Las evidencias señalan primeramente, que los lectores con alto conocimiento del tema pueden formar modelos situacionales incluso cuando los textos presentan baja cohesión, debido a que dicha condición induce al lector a generar mayor inferencias para cubrir los vacíos en el texto (McNamara, Kintsch, Songer \& Kintsch, 1996). Investigaciones posteriores han puesto de manifiesto que el efecto del conocimiento previo en la comprensión, es moderado a su vez tanto por el conocimiento bien estructurado -amplitud y profundidad- que posee el lector, como por el dominio de estrategias de procesamiento que permiten la activación e integración estratégica de éste (McNamara \& O’Reilly, 2010).

2 Desde la Lingüística Sistémico-Funcional, Lemke (1985) plantea una tipología de relaciones intertextuales de la cual no nos ocupamos por considerar que sus características la hacen menos compatible con las otras tradiciones revisadas en este artículo. 